\title{
Surface-electrode decelerator and deflector for Rydberg atoms and molecules
}

\section{Journal Article}

\section{Author(s):}

Allmendinger, Pitt; Deiglmayr, Johannes; Agner, Josef A.; Schmutz, Hansjürg; Merkt, Frédéric

Publication date:

2014-10

Permanent link:

https://doi.org/10.3929/ethz-a-010803696

\section{Rights / license:}

In Copyright - Non-Commercial Use Permitted

Originally published in:

Physical Review A 90(4), https://doi.org/10.1103/PhysRevA.90.043403

\section{Funding acknowledgement:}

149216 - Zeeman and Stark deceleration of atoms and molecules (SNF) 
This article may be downloaded for personal use only. Any other use requires prior permission of the author and The American Physical Society (APS).

The following article appeared in Phys. Rev. A 90, 043403 (2014) and may be found at https://doi.org/10.1103/PhysRevA.90.043403. 


\title{
Surface-electrode decelerator and deflector for Rydberg atoms and molecules
}

\author{
P. Allmendinger, J. Deiglmayr, J. A. Agner, H. Schmutz and F. Merkt \\ Laboratorium für Physikalische Chemie, ETH Zürich, CH-8093, Switzerland
}

(Dated: November 19, 2019)

\begin{abstract}
A surface-electrode decelerator and deflector for Rydberg atoms and molecules has been developed with the goal of performing collisional experiments. Translationally cold $\mathrm{H}_{2}$ molecules in a supersonic beam were excited to Rydberg-Stark states of principal quantum number $n=31$, loaded into electric traps moving at constant speed above the surface of a bent printed circuit board, and deflected from the original direction of the supersonic beam by an angle of $10^{\circ}$. The phase-space characteristics of the deflected beam were characterized by measuring the time-of-flight distribution and images of the Rydberg molecules and comparing them to the results of numerical particle-trajectory simulations. More than $1000 \mathrm{H}_{2}$ molecules were deflected per experimental cycle at a repetition rate of $25 \mathrm{~Hz}$. The phase-space characteristics of the deflector make it attractive to study ion-molecule reactions at low collision energies.
\end{abstract}

PACS numbers: 33.80.Rm, 37.10.De

\section{INTRODUCTION}

Supersonic beams are an important tool to study the properties of isolated atoms and molecules in the gas phase [1]. They provide an almost collision-free environment and enable one to cool internal and translational degrees of freedom of a molecular sample to temperatures of the order of $1 \mathrm{~K}$. The high degree of directionality that can be achieved when a supersonic beam is collimated by a skimmer further provides almost ideal conditions for high-resolution spectroscopic experiments and for studies of elastic, inelastic and reactive collisions between gas particles.

With the demonstration of multistage Stark deceleration 15 years ago [2], a new range of experiments with supersonic beams has been initiated which rely on the ability to continuously tune the velocity of supersonic beams of polar molecules and slow the beams to very low velocities, even below $100 \mathrm{~m} / \mathrm{s}$. This ability offers new opportunities for high-resolution spectroscopic experiments and studies of collisions at low temperature [3]. Since 1999, the range of methods and instruments with which velocity-tunable beams of atoms and molecules can be generated has been considerably widened [4-6]. Today, velocity-tunable beams of paramagnetic atoms and molecules can be generated by multistage Zeeman deceleration [7-11], and Rydberg-Stark deceleration can be used to slow-down supersonic beams of any atom or molecule that can be excited to long-lived Rydberg states [12-14]. Miniaturized, on-chip versions of the decelerators have been developed $[15,16]$ and so have decelerators based on moving traps in free space [17-21], on alternating field gradients [22], and on optical [23] and microwave [24] fields.

When studying collisions using velocity-tunable supersonic beams, the fact that only a small fraction of all atoms or molecules in the beam is decelerated can pose problems. Indeed, collisions with the nondecelerated particles in the beam, e.g., the carrier gas or atoms and molecules with quantum states or velocities and initial positions outside the acceptance of the decelerator, are difficult to avoid and can significantly complicate the data acquisition and analysis. To circumvent these difficulties, decelerators have been designed with which the atom and molecule samples can be deflected or guided away from the original propagation axis of the supersonic beam during or after deceleration [25-28]. Recently, Narevicius and coworkers have demonstrated that reactive scattering can be studied at very low collision energies if two supersonic beams of almost the same, but not necessarily a low, velocity are merged using a magnetic guide to deflect one of the beams [29]. Similar experiments have also been performed by merging both beams using a magnetic guide for one beam and an electric guide for the other [30].

We present here a surface-electrode decelerator and deflector for beams of Rydberg atoms and molecules. The device can be used to merge two supersonic beams, one of which contains Rydberg atoms or molecules, and adjust their relative velocity. The deflection further enables one to eliminate undesired collisions with nondecelerated particles. The Rydberg electron in the Rydberg-Stark states used for deflection is almost entirely decoupled from the ion core and orbits around it at distances much larger than those at which typical ion-molecule reactions take place. Consequently, it does not influence the outcome of an ion-molecule reaction [31,32]. Being able to deflect and control the velocity of Rydberg-atom or molecule beams should therefore enable the study of ion-molecule reactions at temperatures below $1 \mathrm{~K}$. The characterization of the surface-electrode deflector described in this article represents a first step toward this goal. To illustrate its operational principles we have chosen molecular hydrogen $\left(\mathrm{H}_{2}\right)$, a nonpolar, diamagnetic molecule which is notoriously difficult to produce at low temperature and to manipulate with electromagnetic fields. 


\section{EXPERIMENT}

The experiments were carried out using the same laser systems as used in our previous experiments on the deceleration and trapping of hydrogen molecules in Rydberg-Stark states and described in Ref. [33], to which we refer for details. This section focuses on the aspects of the experimental procedure and setup that were modified for the present investigation and which concern primarily the Rydberg-Stark deflector depicted schematically in Fig. 1.

The $\mathrm{H}_{2}$ molecules in a supersonic beam were photoexcited to low-field-seeking $n k m$ Rydberg-Stark states with an $\mathrm{H}_{2}^{+}$ion core in its ground rovibronic state $(n$ is the principal quantum number, $k$ the difference between the parabolic quantum numbers $n_{1}$ and $n_{2}$, and $m$ the magnetic quantum number [34]; the electric dipole moment of a Rydberg-Stark state is $3 n k e a_{0} / 2$ ) using the resonant three-photon excitation sequence

$$
n k m\left[\mathrm{X}^{+2} \Sigma_{\mathrm{g}}^{+}(0,0)\right] \leftarrow \mathrm{I}^{1} \Pi_{\mathrm{g}}(0,2) \leftarrow \mathrm{B}^{1} \Sigma_{\mathrm{u}}^{+}(3,1) \leftarrow \mathrm{X}^{1} \Sigma_{\mathrm{g}}^{+}(0,0),
$$

in which the numbers in parentheses represent the vibrational and rotational quantum numbers of the molecular states. The force

$$
\vec{F}=\nabla\left(\vec{\mu}_{\mathrm{el}} \cdot \vec{E}\right)
$$

resulting from the interaction of the electric dipole moment $\vec{\mu}_{\mathrm{el}}$, having a magnitude up to 3000 Debye at $n=30$, with tailored electric-field gradients is used to manipulate the velocity distribution of the hydrogen molecules.

The pulsed VUV (B - X transition), visible (I - B transition) and near-IR ( $k k m$ - I transition) lasers employed in the successive steps of Equation (1) were described in Ref. [26]. The pulsed supersonic beam of molecular hydrogen was generated by expanding pure $\mathrm{H}_{2}$ in vacuum from a reservoir held at a pressure of 2 bar using a pulsed valve. To reduce the initial velocity of the beam to about $1300 \mathrm{~m} / \mathrm{s}$, the body of the valve was cooled to $77 \mathrm{~K}$ by connecting it to a cold finger filled with liquid nitrogen. The supersonic beam was collimated with a skimmer before entering the photoexcitation region. The photoexcitation was carried out $31 \mathrm{~cm}$ downstream from the pulsed valve between two parallel metallic plates (region (i) in Fig. 1) used to generate a spatially homogeneous electric field of $75 \mathrm{~V} / \mathrm{cm}$ at the point where the supersonic beam crossed the laser beams at right angles. The frequency of the near-IR laser was set so as to excite narrow distributions of low-field-seeking $m=3$ Rydberg-Stark states.

The actual deflector (region (ii) in Fig. 1) is located $5 \mathrm{~mm}$ away from the photoexcitation spot along the propagation axis of the supersonic beam. It consists of a 5-cm-long printed circuit board containing 50 equidistant copper electrodes. Applying precalculated electric potentials to these electrodes enables one to generate tubular electric traps above the surface, as explained in Ref. $[16,35]$. The first $4 \mathrm{~mm}$ of the deflector surface are parallel to the $Z$ axis and enable an easy loading of the hydrogen molecules into the moving traps. The next $42.5 \mathrm{~mm}$ form an arc of a circle with a curvature radius of $244 \mathrm{~mm}$, corresponding to a deflection angle of $10^{\circ}$. The final $3.5 \mathrm{~mm}$ are parallel to the axis of the deflected beam ( $Z^{\prime}$ in Fig. 1). The copper-electrode design was identical to that used in our planar surface-electrode decelerator [35].

Electric traps moving above the decelerator surface at velocities

$$
v=3 d \omega /(2 \pi)
$$

where $d(1 \mathrm{~mm}$ in our experiments) represents the center-to-center spacing between adjacent electrodes, were generated by applying oscillating electric potentials (called waveforms) of the form $V_{i}=(-1)^{i} V_{0}\left[1+\cos \left(\omega t+\phi_{i}\right)\right] / 2$ on the electrodes labeled by the index $i$ running from 1 to 50 . The choice of the oscillation frequency $\omega$ and phases $\phi_{i}=$ $(2-i) 2 \pi / 3[15,16]$ of the waveforms guarantees that the electric traps move at a constant velocity $v$ above the printed circuit board. The peak amplitude $V_{0}$ of the waveforms determines the depth of the moving traps and can be adjusted to optimize the phase-space acceptance of the deflector and the phase-space characteristics of the deflected beam, as explained in more detail in the results section. In our device, the moving electric traps generated by these waveforms are separated by $3 \mathrm{~mm}$ and have a cylindrical shape with a diameter of $0.5 \mathrm{~mm}$ and their axes are parallel to the $Y$ axis. To limit the length of the electric traps to about $4 \mathrm{~mm}$, the surface electrodes are wider near the edge of the deflector where the waveforms are applied via point contacts. Acceleration can be achieved simultaneously to deflection by introducing a linear chirp in the waveform frequency, according to Equation (4) [16]

$$
\omega(t)=\omega_{0}+a \frac{2 \pi}{3 d}\left(t-t_{0}\right)
$$

After the device, the $\mathrm{H}_{2}$ Rydberg molecules fly in free space toward a microchannel-plate (MCP) detector (two plates in chevron configuration) with grounded front plate and a phosphor screen for imaging with a charge-coupled-device 
(CCD) camera (region (iii) in Fig. 1). Ionization of the $\mathrm{H}_{2}$ Rydberg states at the MCP initiates electron cascades in the channels. This configuration enables one to determine the velocity distribution of the deflected molecules along the beam-propagation axis from their flight times between the photoexcitation spot and the MCP front plate. The velocity distributions in the directions perpendicular to the beam propagation axis are extracted from the images of the beams. The quantitative analysis of the velocity distributions is performed by comparing the experimental images and time-of-flight distributions with images and time-of-flight distributions calculated using the particle-trajectory simulation program described in Refs. $[16,36]$. The typical time of flight of the molecules from the photoexcitation spot to the detector is about $80 \mu \mathrm{s}$, which is significantly shorter than their observed decay times [37].

\section{RESULTS}

The lower panel of Fig. 2 presents the results of a series of measurements in which the $\mathrm{H}_{2}$ molecules in $n=31, k=$ $13-17, m=3$ Rydberg-Stark states were guided and deflected at constant velocities using precalculated waveforms of amplitude $V_{0}=80 \mathrm{~V}$. The figure displays the velocity distribution of the deflected molecules obtained by dividing the distance separating the end of the deflector and the detector by the time of free flight between the switch off of the waveforms and detection. The size of the cloud of trapped molecules (radius of about $200 \mu \mathrm{m}$ ) is so small that its effects can be neglected in this analysis. The experimental data are displayed as black traces and are compared to the results of particle-trajectory simulations (red traces). In each case, the central velocity is indicated by a vertical dashed line and corresponds closely to the final velocity the deflector waveforms were programmed for (see Eq. (3)). With a half width at half maximum of about $50 \mathrm{~m} / \mathrm{s}$, the velocity distributions correspond to a temperature of about $300 \mathrm{mK}$ and are broader than the velocity distribution of the original beam represented in the top panel of Fig. 2. The fact that the maxima of the final velocity distributions in the top and bottom traces of Fig. 2b lie slightly outside the initial velocity distribution (Fig. 2a) of the Rydberg molecules further shows that the deflection, in these cases, is accompanied by a slight acceleration or deceleration. Deflected beams with narrower velocity distributions can be obtained by reducing the waveform amplitudes, however, at the expense of a reduced number of deflected molecules.

The images of the $\mathrm{H}_{2}$ molecules presented in Fig. 3a-c were obtained after guiding molecules initially excited to a narrow range of Rydberg-Stark states $(n=31, k=7-11, m=3)$ at a constant velocity of $1290 \mathrm{~m} / \mathrm{s}$ with waveforms of amplitudes of 80,60 and $40 \mathrm{~V} / \mathrm{cm}$, respectively. The spots corresponding to the deflected molecules are located in the lower half of the images and are sharp. In contrast, the signal corresponding to the undeflected molecules is more diffuse and structured, with two maxima along the $Y$ axis on both sides of the position corresponding to the deflected sample. This double-peak structure becomes more and more pronounced as the waveform amplitude is reduced and the missing intensity in the central part of the images can be attributed to the deflected molecules. The spread of the undeflected part of the beam along the $X^{\prime}$ axis is larger than that of the deflected sample. This observation results from the fact that the molecules that are loaded into the trap remain confined in a small volume over the entire length of the printed circuit board, whereas the molecules that are initially located outside the trap volume fly apart and are subject to forces that weakly repel them from the deflector. This effect is most pronounced for the experiments carried out with the largest waveform amplitudes. The images obtained from the particle-trajectory simulations reproduce the main features of the experimental data well, but slightly underestimate the contrast of the double-peak structure of the undeflected beam. The number of deflected molecules decreases by a factor of about 2 upon reduction of the waveform amplitudes from $80 \mathrm{~V}$ to $40 \mathrm{~V}$. A similar behavior is observed when the molecules are excited to Rydberg-Stark states having smaller dipole moments.

The almost perfect agreement between the experimental and simulated velocity distributions (see Fig. 2) and images (see Fig. 3) makes it possible to quantify the efficiency of the deflector. Under the conditions used to record the data presented in Figs. 2 and 3, $20 \%$ of the molecules initially excited to Rydberg-Stark states are deflected when the amplitude of the waveforms is $80 \mathrm{~V}$. This fraction reduces to about $10 \%$, corresponding to about $10^{3}$ molecules, when the amplitude is reduced to $40 \mathrm{~V}$. The main reason for the loss of molecules is the fact that the photoexcitation volume is larger than the volume of the electric traps, as is clearly illustrated by the complementary nature of the distributions of the deflected and undeflected beams along the $Y$ axis in Fig. 3. The electric-field distributions resulting from the applied waveforms are such that, after leaving the deflector, the deflected clouds expand almost freely in the $X^{\prime}$ and $Z^{\prime}$ directions, but are recollimated, and even refocussed for the highest waveform amplitudes in the $Y^{\prime}=Y$ direction, as explained below. Consequently, whereas the velocity distributions of the deflected molecules along the $X^{\prime}$ and $Z^{\prime}$ directions can directly be extracted from the size of the images and the time-of-flight distributions, respectively, no reliable information on the velocity distribution in the $Y^{\prime}$ direction can be extracted from the experimental data.

Panels a and b of Fig. 4 compare the widths of the velocity distributions along the $X^{\prime}$ and $Z^{\prime}$ directions, respectively, obtained from the images (circles) recorded using waveforms of different amplitudes with the velocity distributions 
extracted from the particle-trajectory simulations (squares). Overall, the widths extracted from the experimental data and the particle-trajectory simulations are in good agreement, with the exception of the two points in Fig. $4 \mathrm{~b}$ corresponding to waveform amplitudes of 40 and $50 \mathrm{~V}$, where the widths extracted from the experimental data exceed those predicted by the particle-trajectory simulations. The data indicate a gradual increase of these widths, from about $20 \mathrm{~m} / \mathrm{s}$ to about $40 \mathrm{~m} / \mathrm{s}$ in the $X^{\prime}$ direction and from about $35 \mathrm{~m} / \mathrm{s}$ to $50 \mathrm{~m} / \mathrm{s}$ in the $Z^{\prime}$ direction, as the waveform amplitude increases from 40 to $80 \mathrm{~V}$. This increase indicates a heating of the trapped sample which increases as the moving traps are made deeper. Deflection with low waveform amplitudes therefore yields colder samples, but at the cost of a reduced number of deflected molecules.

Fig. 5 summarizes the main aspects of the evolution of the phase-space distribution obtained from the particletrajectory simulations for waveform amplitudes of $40 \mathrm{~V}$ (a-c) and $80 \mathrm{~V}$ (d-f). In each panel, the black dots on the left-hand-side plot represent the distribution in the $X, Y, Z$ coordinate system immediately after trap loading and those on the right-hand-side plot the distribution in the $X^{\prime}, Y^{\prime}=Y, Z^{\prime}$ coordinate system at the exit of the deflector (see Fig. 1 for the definition of these coordinate systems). The area shaded in gray corresponds to the phase-space area explored over the entire deflection process determined in the instantaneous coordinate system where the $X$ and $Z$ axes are perpendicular and tangential to the surface of the deflector, respectively. These phase-space diagrams reveal that the heating is most significant in the direction perpendicular to the deflector surface, as a result of the trap-loading process and the coupling between the $X$ and $Y$ directions caused by the trap anharmonicity and the deflection, which rotates the coordinate system around the $Y^{\prime}=Y$ axis by $10^{\circ}$. The heating effect is also noticeable in the propagation direction of the moving traps (i.e., tangential to the surface). In the $Y^{\prime}=Y$ direction, which is not affected by the deflection and is characterized by a flat potential, the main effect is a rotation of the phase-space distribution. This distribution starts spiraling when the particles with the highest velocity in $Y$ direction reach the repulsive walls of the tubular traps, at which point the beam is recollimated, as already mentioned in the previous paragraph. The data presented in Fig. 5 provide support to the conclusions derived from the analysis of the time-of-flight distributions (Fig. 2) and images (Fig. 3) and indicate that the heating of the trapped sample is roughly proportional to the waveform amplitude. For amplitudes of $40 \mathrm{~V}(80 \mathrm{~V})$, about $1000(2000) \mathrm{H}_{2}$ molecules in $n=31, k \approx 9 \mathrm{Rydberg}$-Stark states, characterized by a translational temperature of about $200 \mathrm{mK}(300 \mathrm{mK})$ could be deflected per experimental cycle at a repetition rate of $25 \mathrm{~Hz}$.

Larger decelerations than achieved in the bottom trace of Fig. 2b can be achieved by operating the device simultaneously as deflector and decelerator according to Eq. (4), as demonstrated in Fig. 6. In this figure, the velocity distributions obtained after deceleration from an initial velocity of $1300 \mathrm{~m} / \mathrm{s}$ to final velocities of $1100,900,800$ and $700 \mathrm{~m} / \mathrm{s}$ are presented. Whereas the deceleration to $1100 \mathrm{~m} / \mathrm{s}$ is not accompanied by a significant reduction of the phase-space acceptance of the device, deceleration to lower velocities leads to a reduction of the number of detected $\mathrm{H}_{2}$ molecules by a factor of about two and four when the deceleration is carried out to final velocities of 900 and $700 \mathrm{~m} / \mathrm{s}$, respectively. The reduction of phase-space acceptance is accompanied by a narrowing of the final velocity distribution.

\section{CONCLUSIONS}

A surface-electrode decelerator and deflector for Rydberg atoms and molecules made of 50 copper electrodes printed on a bent circuit board has been developed and used to deflect $n=31 \mathrm{H}_{2}$ Rydberg molecules in a supersonic beam by an angle of $10^{\circ}$. The phase-space characteristics of the deflected beam were determined by imaging and time-of-flight techniques. About $1000 \mathrm{H}_{2}$ molecules could be deflected per experimental cycle at a repetition rate of $25 \mathrm{~Hz}$. The temperature of the deflected sample was found to be below $1 \mathrm{~K}$ and could be adjusted by changing the amplitude of the oscillating electric potentials applied to the surface electrodes. Introducing a linear chirp in the frequency of the oscillating electric potential enabled us to decelerate the beam of Rydberg molecules during deflection, and thus achieve a wider range of final velocities.

The low translational temperature of the deflected beam, the flux of more than $10^{4}$ Rydberg $\mathrm{H}_{2}$ molecules per second and square millimeter, the broad range of final velocities, and the fact that the Rydberg states can be prepared with an ion core in a selected rovibronic level (the $\mathrm{X}{ }^{2} \Sigma_{g}^{+}\left(v^{+}=0, N^{+}=0\right)$ state for the experiments presented in this article) makes the device a promising tool for the study of ion-molecule reactions at low collision energies. The method can be applied to deflect beams of heavier species than molecular hydrogen and to deflect beams of molecular hydrogen by larger angles. The maximal degree of curvature the $\mathrm{H}_{2}$ Rydberg molecules can follow and the maximum mass of the molecules that can be deflected by a given angle depend on the magnitude of the electric potentials applied to the surface electrodes, on the initial velocity of the supersonic beam, and on the lifetime of the Rydberg states. Assuming an initial velocity of $385 \mathrm{~m} / \mathrm{s}$ typical for a supersonic beam with krypton as a carrier gas and lifetimes $\tau$ between 
$100 \mu \mathrm{s}$ and $300 \mu \mathrm{s}$ for the Rydberg Stark states used in our experiments [37], the maximum decelerator length is between 3.8 and $11.5 \mathrm{~cm}$. Considering the centrifugal potential (see Hogan et al. [38]) for a waveform amplitude of $80 \mathrm{~V}$, an $n=30, k=27$ Stark state and a minimal trap depth of $1 \mathrm{~K}$, we can derive the maximal deflection angle in dependence of the mass of the Rydberg atom or molecule. We find an inverse dependence of the maximal angle on the mass. For lifetimes of $100 \mu \mathrm{s}$ and $300 \mu \mathrm{s}$, the maximal deflection angle is $10^{\circ}$ for a mass of $180 \mathrm{u}(\tau=100 \mu \mathrm{s})$ and $540 \mathrm{u}((\tau=300 \mu \mathrm{s})$. We also conclude that particles could even complete a full circle if their mass is less than $5 \mathrm{u}$ $(\tau=100 \mu \mathrm{s})$ and less than $18 \mathrm{u}(\tau=300 \mu \mathrm{s})$.

\section{Acknowledgments}

This work was supported financially by the Swiss National Science Foundation (Project No. 200020-149216) and the European Research Council advanced grant program (Project No. 228286).

[1] G. Scoles (ed.), Atomic and molecular beam methods, Oxford University Press, New York (1988-1992), 2 vols.

[2] H. L. Bethlem, G. Berden and G. Meijer, Phys. Rev. Lett. 83, 1558 (1999).

[3] M. Kirste, X. Wang, H. C. Schewe, G. Meijer, K. Liu, A. van der Avoird, L. M. C. Janssen, K. B. Gubbels, G. C. Groenenboom and S. Y. T. van de Meerakker, Science 338, 1060 (2012).

[4] S. D. Hogan, M. Motsch and F. Merkt, Phys. Chem. Chem. Phys. 13, 18705 (2011).

[5] S. Y. T. van de Meerakker, H. L. Bethlem, N. Vanhaecke and G. Meijer, Chem. Rev. 112, 4828 (2012).

[6] E. Narevicius and M. G. Raizen, Chem. Rev. 112, 4879 (2012).

[7] N. Vanhaecke, U. Meier, M. Andrist, B. H. Meier and F. Merkt, Phys. Rev. A 75, 031402(R) (2007).

[8] E. Narevicius, A. Libson, C. G. Parthey, I. Chavez, J. Narevicius, U. Even and M. G. Raizen, Phys. Rev. A 77, 051401 (2008).

[9] A. W. Wiederkehr, H. Schmütz, M. Mötsch and F. Merkt, Mol. Phys. 110, 1807 (2012).

[10] T. Momose, Y. Liu, S. D. Zhou, P. Djuricanin and D. Carty, Phys. Chem. Chem. Phys. 15, 1772 (2013).

[11] M. Motsch, P. Jansen, J. A. Agner, H. Schmutz and F. Merkt, Phys. Rev. A 89, 043420 (2014).

[12] S. R. Procter, Y. Yamakita, F. Merkt and T. P. Softley, Chem. Phys. Lett. 374, 667 (2003).

[13] E. Vliegen, H. J. Wörner, T. P. Softley and F. Merkt, Phys. Rev. Lett. 92, 033005 (2004).

[14] S. D. Hogan, Ch. Seiler and F. Merkt, Phys. Rev. Lett. 103, 123001 (2009).

[15] S. A. Meek, H. Conrad and G. Meijer, Science 324, 1699 (2009).

[16] S. D. Hogan, P. Allmendinger, H. Sassmannshausen, H. Schmutz and F. Merkt, Phys. Rev. Lett. 108, 063008 (2012).

[17] E. Vliegen, S. D. Hogan, H. Schmutz and F. Merkt, Phys. Rev. A 76, 023405 (2007).

[18] S. D. Hogan and F. Merkt, Phys. Rev. Lett. 100, 043001 (2008).

[19] A. Osterwalder, S. A. Meek, G. Hammer, H. Haak and G. Meijer, Phys. Rev. A 81, 051401(R) (2010).

[20] A. Trimeche, M. N. Bera, J.-P. Cromières, J. Robert and N. Vanhaecke, Eur. Phys. J. D 65, 263 (2011).

[21] E. Lavert-Ofir, S. Gersten, A. B. Henson, I. Shani, L. David, J. Narevicius and E. Narevicius, New J. of Phys. 13, 103030 (2011).

[22] K. Wohlfart, F. Filsinger, F. Grätz, J. Küpper and G. Meijer, Phys. Rev. A 78, 033421 (2008).

[23] R. Fulton, A. I. Bishop and P. F. Barker, Phys. Rev. Lett. 93, 243004 (2004).

[24] S. Merz, N. Vanhaecke, W. Jäger, M. Schnell and G. Meijer, Phys. Rev. A 85, 063411 (2012).

[25] C. E. Heiner, H. L. Bethlem and G. Meijer, Chem. Phys. Lett. 473, 1 (2009).

[26] Ch. Seiler, S. D. Hogan, H. Schmutz, J. A. Agner and F. Merkt, Phys. Rev. Lett. 106, 073003 (2011).

[27] P. Lancuba and S. D. Hogan, Phys. Rev. A 88, 043427 (2013).

[28] H. Ko and S. D. Hogan, Phys. Rev. A 89, 053410 (2014).

[29] A. B. Henson, S. Gersten, Y. Shagam, J. Narevicius and E. Narevicius, Science 338, 234 (2012).

[30] B. Bertsche, J. Jankunas and A. Osterwalder, Chimia 68, 256 (2014).

[31] S. T. Pratt, J. L. Dehmer, P. M. Dehmer and W. A. Chupka, J. Chem. Phys. 101, 882 (1994).

[32] M. Matsuzawa, Phys. Rev. A 82, 054701 (2010).

[33] Ch. Seiler, S. D. Hogan and F. Merkt, Phys. Chem. Chem. Phys. 13, 19000 (2011).

[34] T. F. Gallagher, Rydberg Atoms, Cambridge University Press, Cambridge (1994).

[35] P. Allmendinger, J. A. Agner, H. Schmutz and F. Merkt, Phys. Rev. A 88, 043433 (2013).

[36] E. Vliegen, P. Limacher and F. Merkt, Eur. Phys. J. D 40, 73 (2006).

[37] C. Seiler, S. D. Hogan and F. Merkt, Chimia 66, 208 (2012).

[38] S. D. Hogan, Ch. Seiler and F. Merkt, J. Phys. B: At. Mol. Opt. Phys. 46, 045303 (2013). 


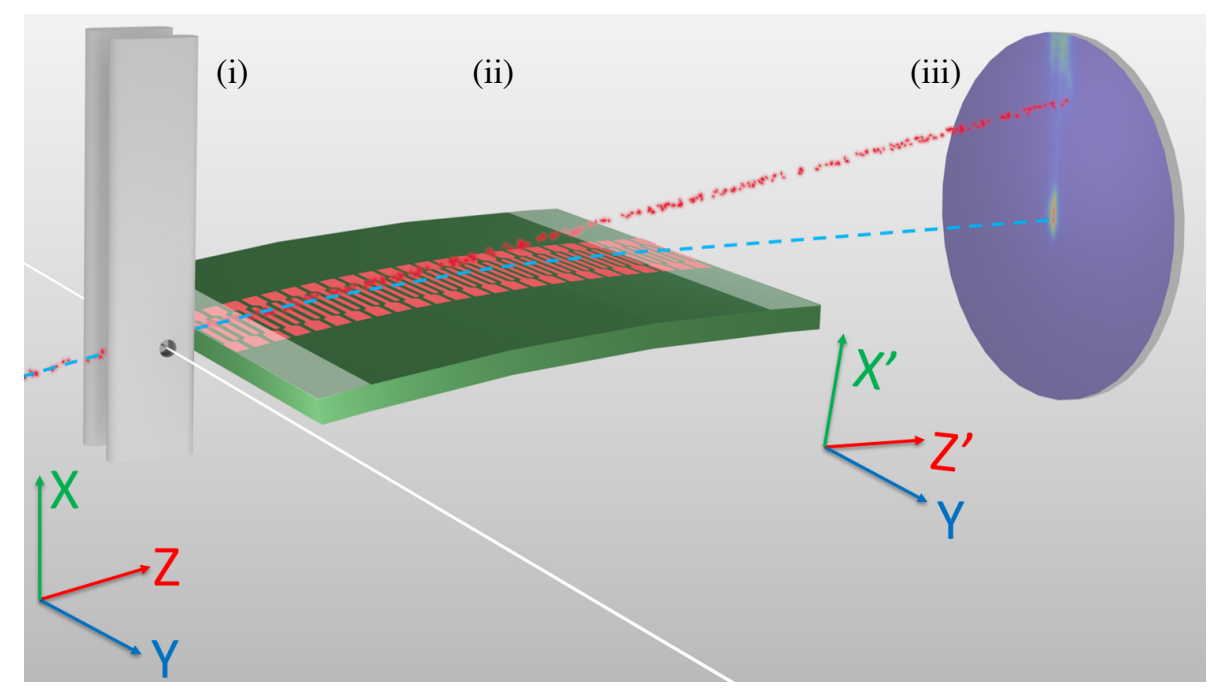

FIG. 1: (Color online) Schematic representation of the surface-electrode deflector used to deviate $\mathrm{H}_{2}$ Rydberg molecules in a supersonic beam by $10^{\circ}$ from the initial beam-propagation axis. (i) Photoexcitation region where the Rydberg molecules are prepared in the homogeneous electric field generated by applying dc electric potential to two parallel planar electrodes separated by $4 \mathrm{~mm}$. (ii) 50-mm-long printed circuit board with 50 equidistant copper electrodes. The first 4-mm-long and the last 3.5-mm-long sections along the beam propagation axis are planar whereas the middle 42.5-mm-long section forms an arc of a circle with a radius of $244 \mathrm{~mm}$. (iii) MCP detector with anode connected to a phosphor screen and a CCD camera for imaging. 
(a)

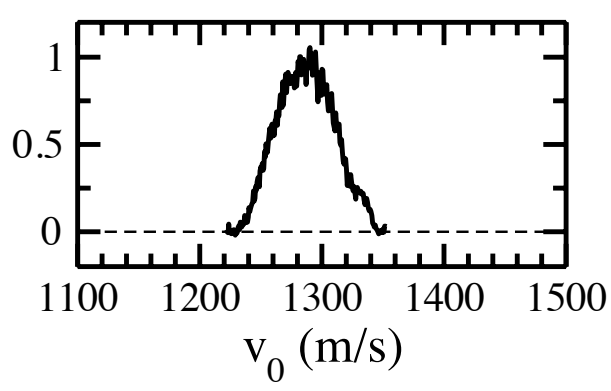

(b)

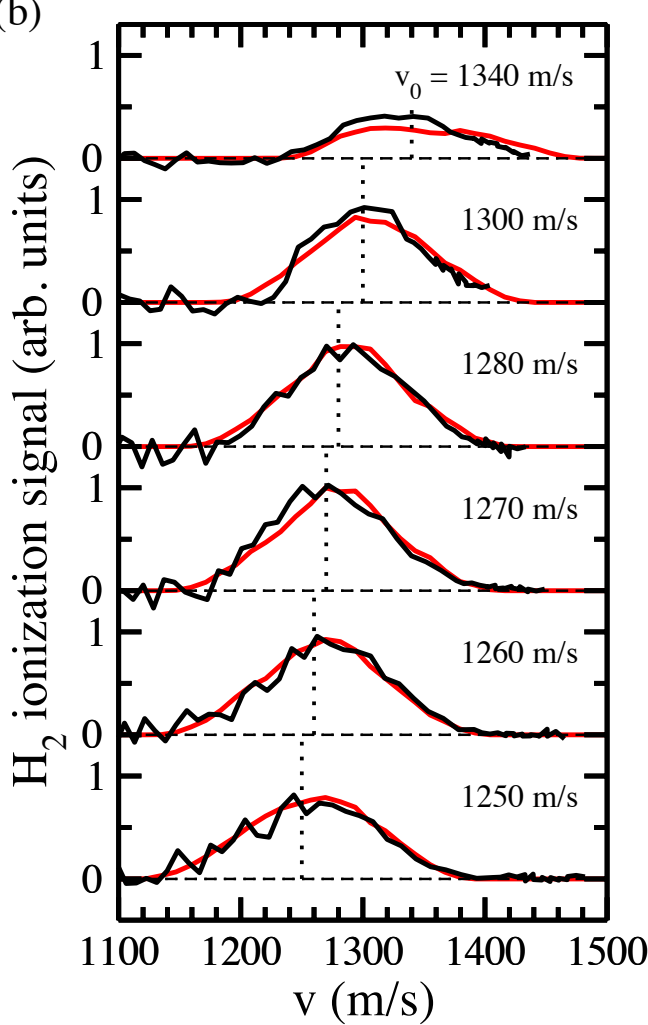

FIG. 2: (Color online) (a) Longitudinal velocity distribution of the beam obtained without applying any waveforms to the surface electrodes. (b) Longitudinal velocity distributions of the deflected $\mathrm{H}_{2}$ Rydberg molecules $((n=31, k=13-17))$ guided over the chip at velocities $v_{0}$ (determined with Eq. (3)) ranging from $1250 \mathrm{~m} / \mathrm{s}$ to $1340 \mathrm{~m} / \mathrm{s}$ using waveforms with $80 \mathrm{~V}$ amplitude. Black traces: experimental results. Red traces: results of particle-trajectory simulations. 


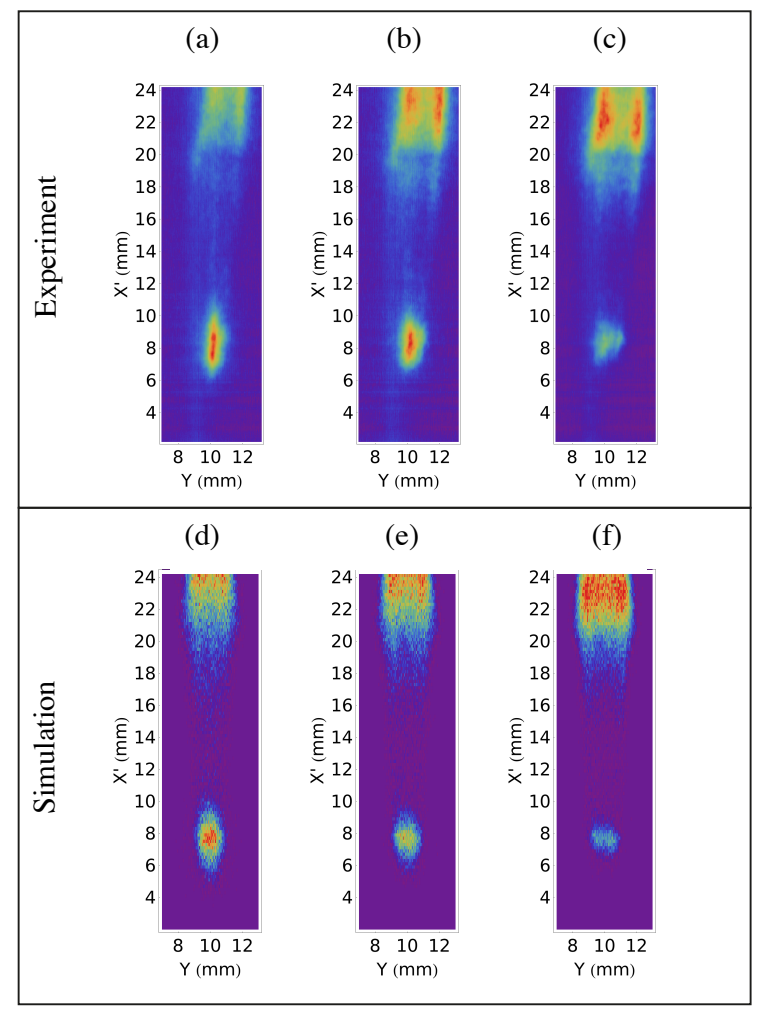

FIG. 3: (Color online) Spatial distribution of the $\mathrm{H}_{2}$ Rydberg molecules $(n=31, k=7-11)$ detected at the imaging MCP detector following deflection at a constant velocity of $1290 \mathrm{~m} / \mathrm{s}$ using waveform amplitudes of (a) $80 \mathrm{~V}$, (b) $60 \mathrm{~V}$ and (c) $40 \mathrm{~V}$. (d)-(e) Corresponding images generated from particle-trajectory simulations. See text for details.
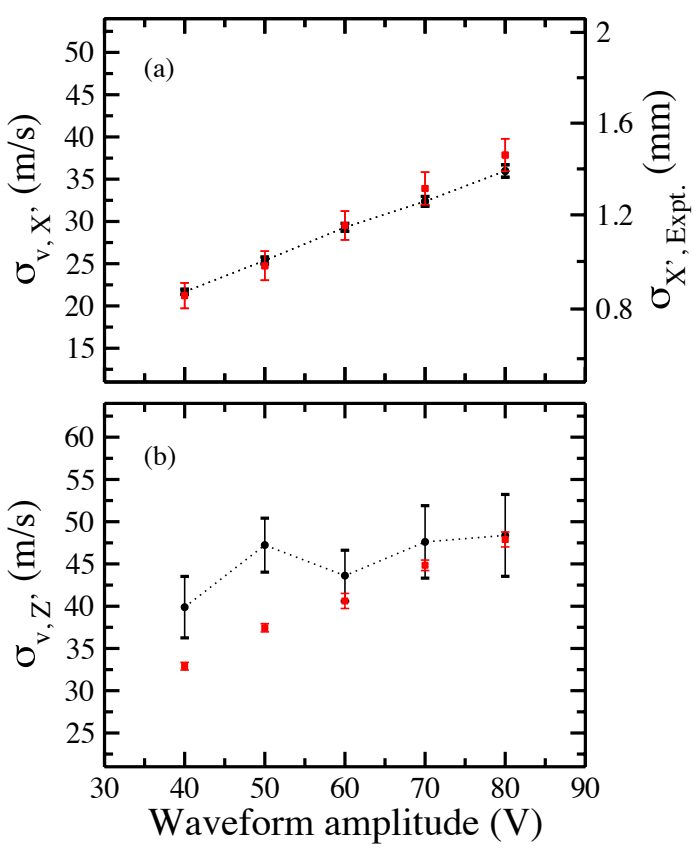

FIG. 4: (Color online) Velocity distributions of the $\mathrm{H}_{2}$ Rydberg molecules $(n=31, k=7-11)$ along the (a) $X^{\prime}$ and (b) $Z^{\prime}$ directions following deflection at a constant velocity of $1290 \mathrm{~m} / \mathrm{s}$ as a function of applied waveform amplitude. The results of particle trajectory simulations are indicated by squares and the experimental results by circles. 


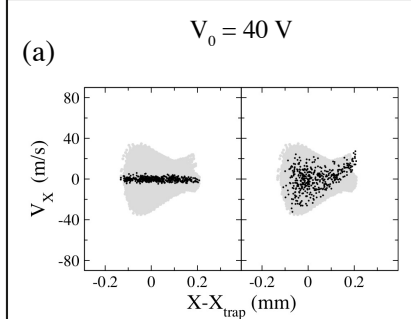

(b)

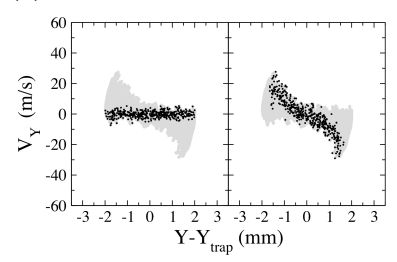

(c)

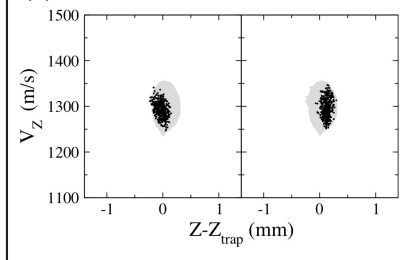

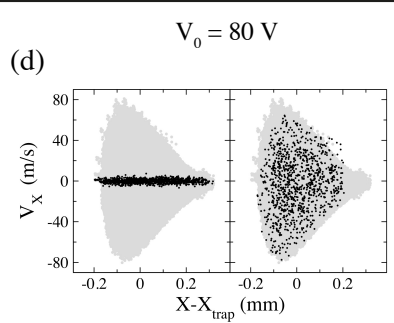

(e)

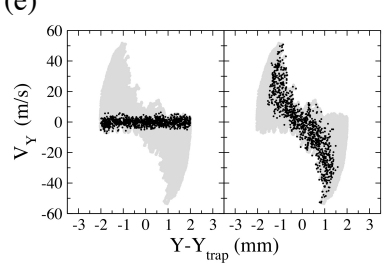

(f)

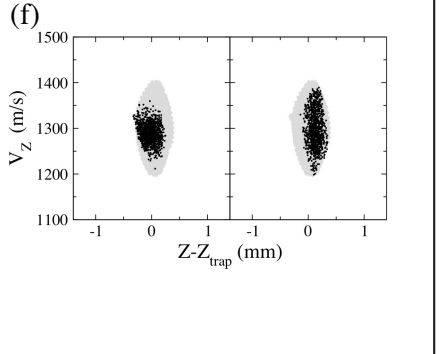

FIG. 5: Phase-space distributions of the deflected $\mathrm{H}_{2}$ Rydberg molecules $(n=31, k=7-11)$ obtained from particletrajectory simulations for waveform amplitudes of (a-c) $40 \mathrm{~V}$ and (d-f) $80 \mathrm{~V}$. The black dots in the left and right parts of each plot correspond to Rydberg molecules at the beginning and the end of the deflector, respectively. The area shaded in gray correspond to the phase-space areas explored over the entire defelction process. The distribution of spatial position is plotted with respect to the center of the trap $\left(X_{\text {trap }}, Y_{\text {trap }}, Z_{\text {trap }}\right)$. 


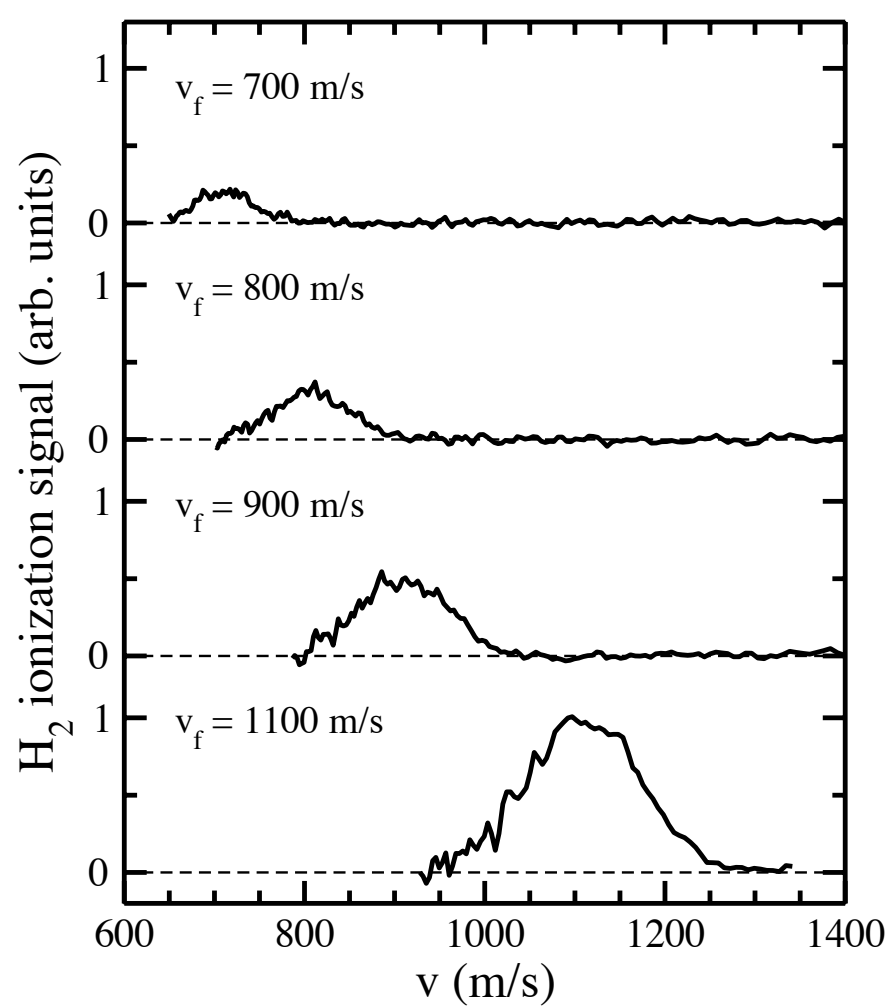

FIG. 6: Velocity distributions obtained after simultaneous deflection a beam of $\mathrm{H}_{2}$ Rydberg molecules $(n=31, k=7-11)$ by $10^{\circ}$ and deceleration from an initial velocity of $1300 \mathrm{~m} / \mathrm{s}$ to final velocities of $1100,900,800$ and $700 \mathrm{~m} / \mathrm{s}$. 